\title{
Erratum to: Pan evaporation and wind run decline in the Cape Floristic Region of South Africa (1974-2005): implications for vegetation responses to climate change
}

\author{
M. Timm Hoffman • Michael D. Cramer • \\ Lindsey Gillson • Michael Wallace
}

Published online: 15 October 2011

(C) Springer Science+Business Media B.V. 2011

\section{Erratum to: Climatic Change (2011) \\ DOI 10.1007/s10584-011-0030-z}

Unfortunately, there are a few errors in the wind run data of this paper. The errors relate to the wind run values and units in the last two columns of Table 2 and a few numbers in sub-section ' 3.3 Wind run'. The corrected text and table are given below. The relevant values are highlighted.

\subsection{Wind run}

Between 1974-2005 wind run (Utotal) decreased significantly at all stations (Tables 2 and 3). There was a $26.9 \%$ decline in average Utotal values for the 20 climate stations over the study period from 2.16 to $1.58 \mathrm{~m} \mathrm{~s}^{-1}$ at an average rate of $0.0188 \pm 0.002 \mathrm{~m} \mathrm{~s}^{-1} \mathrm{a}^{-1}$ (Fig. 2c). Wind run values were greater in the dry summer months than the winter months $\left(2.08 \mathrm{~m} \mathrm{~s}^{-1}\right.$ versus $\left.1.61 \mathrm{~m} \mathrm{~s}^{-1}\right)$ but there was no significant difference in the decline in wind run between the seasons $\left(-0.4003 \% \mathrm{a}^{-1}\right.$ in summer and $-0.5063 \% \mathrm{a}^{-1}$ in winter; $\left.F(1,60)=3.8377, p=0.0548\right)$.

The online version of the original article can be found at doi:http://dx.doi.org/10.1007/s10584-011-0030-z.

M. Timm Hoffman $(\varangle) \cdot$ M. D. Cramer · L. Gillson

Botany Department, University of Cape Town,

Private Bag X3, Rondebosch, 7701, South Africa

e-mail: timm.hoffman@uct.ac.za

M. Wallace

Spatial Analysis Unit, Institute for Resource Utilisation,

Western Cape Department of Agriculture,

Private Bag X1, Elsenburg, 7607, South Africa 
Table 2 Average values and slope of the linear regression line $( \pm \mathrm{SE}$ ) for annual pan evaporation $\left(E_{\text {pan }}\right)$, rainfall $(P)$ and wind run $\left(U_{\text {total }}\right)$ for 20 climate stations in the winter rainfall region of South Africa for the period 1974-2005

\begin{tabular}{|c|c|c|c|c|c|c|}
\hline \multirow[t]{2}{*}{ Station } & $\underline{E_{\text {pan }}}$ & $\mathrm{d}\left(E_{\text {pan }}\right) / \mathrm{d} t$ & $P$ & $\mathrm{~d}(P) / \mathrm{d} t$ & $U_{\text {total }}$ & $\mathrm{d}\left(U_{\text {total }}\right) / \mathrm{d} t$ \\
\hline & $\mathrm{mm} \mathrm{a}^{-1}$ & $\mathrm{~mm} \mathrm{a}^{-2}$ & $\mathrm{~mm} \mathrm{a}^{-1}$ & $\mathrm{~mm} \mathrm{a}^{-2}$ & $\mathrm{~m} \mathrm{~s}^{-1}$ & $\mathrm{~m} \mathrm{~s}^{-1} \mathrm{a}^{-1}$ \\
\hline Aan-de-doorns & 2103 & $-6.2 \pm 2.4$ & 260 & $-2.1 \pm 1.5$ & 2.32 & $-0.025 \pm 0.003$ \\
\hline Boontjieskraal & 1681 & $1.3 \pm 2.1$ & 418 & $1.1 \pm 1.6$ & 1.76 & $-0.018 \pm 0.002$ \\
\hline De Doorns (NIWW) & 2015 & $-16.6 \pm 2.1$ & 337 & $-0.3 \pm 2.1$ & 1.23 & $-0.015 \pm 0.002$ \\
\hline Graafwater Ko-op & 2015 & $-\mathbf{1 7 . 1} \pm \mathbf{3 . 5}$ & 281 & $-0.9 \pm 1.4$ & 1.01 & $-0.010 \pm 0.004$ \\
\hline H.L.S. Boland & 2048 & $-5.8 \pm 2.6$ & 517 & $0.3 \pm 2.3$ & 1.74 & $-0.025 \pm 0.003$ \\
\hline Ideal Hill & 2465 & $-8.9 \pm 4.3$ & 284 & $-1.4 \pm 1.6$ & 2.06 & $-0.011 \pm 0.003$ \\
\hline Jonaskraal & 1892 & $-7.2 \pm 2.7$ & 395 & $0.6 \pm 1.8$ & 2.56 & $-0.008 \pm 0.004$ \\
\hline Klawer Wynkelder & 2549 & $-7.8 \pm 3.6$ & 211 & $-0.8 \pm 1.3$ & 1.83 & $-0.018 \pm 0.006$ \\
\hline La Plaisante & 1909 & $-14.5 \pm 1.8$ & 619 & $-3.7 \pm 3.0$ & 1.53 & $-0.015 \pm 0.005$ \\
\hline Langgewens (WRS) & 2206 & $-5.4 \pm 2.4$ & 409 & $-2.2 \pm 2.0$ & 2.43 & $-0.018 \pm 0.003$ \\
\hline Mooreesburg Ko-op & 2313 & $-3.5 \pm 2.9$ & 410 & $-2.4 \pm 1.8$ & 2.10 & $-0.005 \pm 0.002$ \\
\hline Nortier (WRS) & 1865 & $-17.7 \pm 3.4$ & 220 & $0.0 \pm 1.3$ & 2.45 & $-0.04 \pm 0.004$ \\
\hline Outeniqua (WRS) & 1290 & $-\mathbf{1 0 . 0} \pm \mathbf{1 . 2}$ & 740 & $2.5 \pm 4.2$ & 1.73 & $-0.014 \pm 0.004$ \\
\hline Philadelphia Polisie & 1745 & $-4.4 \pm 3.9$ & 478 & $-1.8 \pm 2.2$ & 1.28 & $-0.017 \pm 0.002$ \\
\hline Porterville Ko-op & 1998 & $-2.6 \pm 2.3$ & 520 & $-2.4 \pm 2.2$ & 1.15 & $-0.017 \pm 0.003$ \\
\hline Prospect & 1855 & $-9.4 \pm 2.3$ & 280 & $-0.9 \pm 1.8$ & 1.86 & $-0.008 \pm 0.003$ \\
\hline Protem & 2026 & $-7.2 \pm 3.5$ & 449 & $2.7 \pm 1.9$ & 2.48 & $-0.044 \pm 0.003$ \\
\hline Riversdal Ko-op & 1682 & $-\mathbf{1 5 . 8} \pm \mathbf{3 . 8}$ & 452 & $-0.2 \pm 2.0$ & 1.47 & $-0.016 \pm 0.003$ \\
\hline Serjeantsrivier & 1634 & $-9.8 \pm 3.1$ & 542 & $1.7 \pm 2.2$ & 1.84 & $-0.032 \pm 0.007$ \\
\hline Veldreserwe & 2272 & $-12.5 \pm 3.1$ & 263 & $-1.3 \pm 1.5$ & 2.09 & $-0.021 \pm 0.002$ \\
\hline
\end{tabular}

Significant trends $(p<0.05)$ are indicated in bold 